\title{
Presencia de anticuerpos frente a Rotavirus en granjas porcícolas semitecnificadas de Cundinamarca, Colombia
}

\author{
Presence of antibodies against Rotavirus in semi-technical pig farms in \\ Cundinamarca, Colombia
}

\begin{abstract}
Adriana Pulido-Villamarín ${ }^{1,6}$, Rubiela Castañeda-Salazar', Kelly Johana MendezCarranza $^{1}$, Astrid Natalia Santamaría-Durán ${ }^{1}$, Ana Karina Carrascal-Camacho ${ }^{2}$, Ricardo Cubillos-Azcárate ${ }^{3,4}$, Corina Zambrano-Moreno ${ }^{5}$
\end{abstract}

\section{RESUMEN}

\begin{abstract}
El objetivo del presente trabajo fue determinar la presencia de anticuerpos frente a rotavirus en sueros de porcinos obtenidos de granjas semitecnificadas de Cundinamarca, Colombia. Se procesaron 170 muestras de varios grupos etarios, utilizando el estuche de ELISA para diagnóstico Ingezim Rotavirus Porcino $\left(\right.$ Ingenasa $^{\circledR}$ ). Se detectó la presencia de anticuerpos frente a Rotavirus en el $78.82 \%$ de las muestras. Se concluye que los animales pudieron haber estado en contacto con el virus en algún momento de su ciclo productivo, lo que sugiere la necesidad de mejoras en el control y prevención de la infección.
\end{abstract}

Palabras clave: seroprevalencia, Rotavirus, porcinos, zoonosis, Colombia

1 Semillero Enfermedades Infecciosas Veterinarias y Zoonosis, Unidad de Investigaciones Agropecuarias - UNIDIA-, Departamento de Microbiología, Facultad de Ciencias, Pontificia Universidad Javeriana, Bogotá, Colombia

${ }^{2}$ Laboratorio de Microbiología de Alimentos, Grupo de Biotecnología Ambiental e Industrial - GBAI Departamento de Microbiología, Facultad de Ciencias, Pontificia Universidad Javeriana, Bogotá, Colombia

${ }^{3}$ Facultad de Medicina Veterinaria, Universidad de Ciencias Aplicadas y Ambientales (UDCA), Bogotá, Colombia

${ }^{4}$ Área Técnica, Asociación Colombiana de Porcicultores (PorkColombia), Colombia

5. Centro de Investigación y Transferencia de Tecnología del Sector Porcícola - CENIPORCINO, Asociación Colombiana de Porcicultores (PorkColombia) - Fondo Nacional de la Porcicultura, Bogotá, Colombia

${ }^{6}$ E-mail: adriana.pulido@javeriana.edu.co

Recibido: 23 de octubre de 2019

Aceptado para publicación: 9 de junio de 2020

Publicado: 11 de agosto de 2020 
The aim of this study was to determine the presence of antibodies against rotavirus in pig sera obtained from semi-technical farms in Cundinamarca, Colombia. For this, 170 samples from various age groups were processed, using the Porcine Ingezim Rotavirus (Ingenasa ${ }^{\circledR}$ ) diagnostic ELISA kit. The presence of antibodies against Rotavirus was detected in $78.82 \%$ of the samples. It is concluded that the animals may have been in contact with the virus at some point in their production cycle, which suggests the need for improvements in the control and prevention of infection.

Key words: seroprevalence, Rotavirus, pigs, zoonosis, Colombia

\section{INTRODUCCIÓN}

La industria porcícola colombiana ha evidenciado en los últimos 10 años un notable y constante crecimiento. En el primer trimestre de 2019, el sacrificio de ganado porcino fue de 1097420 cabezas, comparado con las 995527 cabezas para el mismo periodo del año anterior (DANE, 2019). Asimismo, el consumo per cápita se ha incrementado hasta casi duplicarse en la última década, llegando a los $9.3 \mathrm{~kg} /$ habitante (Maya, 2018).

A lo largo de la cadena productiva porcícola, se debe asegurar la calidad de los productos destinados al consumo humano mediante la implementación de programas de mejoramiento y fortalecimiento de las medidas de bioseguridad, manejo y sanidad (Porkcolombia, 2014). La presencia de patógenos, especialmente aquellos que producen alteraciones gastrointestinales, ocasionan importantes pérdidas económicas en la industria porcícola, además de constituir un riesgo para la salud pública dado su potencial zoonótico. Entre estos microor-ganismos se encuentran bacterias, parásitos, hongos y virus como Astrovirus, Rotavirus del grupo A, Norovirus y Virus de la Hepatitis E (Monini et al., 2015).
Rotavirus es un virus desnudo de forma icosaédrica cuya cápside está constituida por tres capas proteicas. La cápside externa está conformada por las proteínas estructurales VP7 y VP4, la intermedia por VP6 y la nucleocápside por VP2 (Long y McDonald, 2012). Su genoma se encuentra compuesto por 11 genes que forman una cadena doble de RNA (dsRNA) segmentado y que codifican seis proteínas estructurales (VP1-VP4 y VP6-VP7) y cinco proteínas no estructurales (NSP1-NSP5/NSP6), dentro de las cuales se encuentra la proteína NSP4, primera enterotoxina viral descrita, que consiste en una glicoproteína constituida por 175 residuos de aminoácidos cuyo papel es importante en la morfogénesis y la patogenicidad del virus (Saurabh et al., 2018). Ocho grupos de rotavirus (A-H) han sido caracterizados, donde el grupo A (RVA) es el más comúnmente encontrado en humanos (Bucardo y Nordgren, 2015), siendo los genotipos G1, G2, G3, G4, G9, P[8], P[4] y P[6] los más prevalentes a nivel mundial (Yang et al., 2008). En Colombia, los genotipos más frecuentemente reportados en humanos son G3P8 (33\%), G2P4 (26\%) y G1P8 (25\%) (De la Hoz et al., 2010). Por otro lado, los rotavirus pertenecientes a los grupos A, B, C y E pueden también afectar cerdos, siendo el último detectado únicamente en porcinos (Martella et al., 2010). 
Clínicamente, Rotavirus es un patógeno entérico causante de diarrea acuosa aguda en diferentes especies hospederas (Yoodmeklin et al., 2017). En el humano, la enfermedad diarreica aguda (EDA) es considerada como la causante de más de 400000 muertes y hospitalizaciones por año en niños menores de cinco años a nivel mundial (WHO, 2017). Si bien, dicha enfermedad se asocia con diferentes agentes causales, los virus son los agentes etiológicos de mayor distribución, ocasionando entre el 70 al 80\% de casos en niños dentro del grupo de riesgo, siendo rotavirus el más representativo de ellos (Blanco y Reyes, 2015).

A pesar de que la información sobre la epidemiología o la diversidad antigénica de RVA en cerdos es escasa, se le ha detectado en animales entre los 7 y 60 días generalmente, con alta prevalencia entre las 3 y 5 semanas de edad. A su vez, se destacan los genotipos de mayor frecuencia G3-G5, G9 y G11 combinado con P[5]-[7], P[13], P[23], $\mathrm{P}$ [27] y $\mathrm{P}$ [34] (Vidal et al., 2018). Aunque los virus pertenecientes a este grupo son generalmente aceptados como patógenos específicos de especie, la transmisión interespecie ha sido reportada. Por ejemplo, algunas cepas de RVA pueden ser detectadas en humanos y cerdos, producto de la transmisión del virus de origen animal al humano (Midgley et al., 2012; Malasao et al., 2018).

En algunos países de Latinoamérica como Brasil, Argentina y Paraguay, cepas inusuales de rotavirus G5 han sido detectadas en niños, comprobándose mediante técnicas moleculares, que son producto de un reordenamiento entre virus humanos y porcinos (Martella et al., 2010), de allí que la determinación indirecta de la presencia del virus en granjas porcícolas permite realizar ajustes a los programas de control y prevención, con el fin de evitar los efectos negativos para la producción, derivados de las pérdidas económicas asociadas a la disminución de los índices zootécnicos así como a las posibles implicaciones en salud pública.

\section{Materiales y Métodoos}

Con base a la participación voluntaria de los propietarios de las granjas porcícolas, se realizó un muestreo por conveniencia, colectándose al azar 170 muestras sanguíneas de porcinos clínicamente sanos, distribuidos en seis granjas semitecnificadas de ciclo completo. Estas granjas tienen menos de 400 hembras de cría y sus planes vacunales y sanitarios se manejaban de acuerdo con su situación y según criterio técnico-clínico veterinario. Estaban ubicadas en los municipios Ricaurte, La Vega, Nocaima, Bituima, Zipaquirá y Sasaima, del departamento de Cundinamarca, Colombia.

Las muestras de sangre fueron colectadas de la vena yugular en tubos vacutainer sin anticoagulante (Framstad et al., 2000; Casas, 2013). Las muestras fueron refrigeradas y transportadas en nevera de poliestireno expandido («icopor») hasta los laboratorios de la Pontificia Universidad Javeriana. Las muestras fueron centrifugadas a $1500 \mathrm{~g}$ por 5 minutos, para la obtención del suero. Los sueros fueron procesados mediante la técnica de ELISA indirecta utilizando el estuche Ingezim Rotavirus Porcino (Ingenasa ${ }^{\circledR}$ ), siguiendo las indicaciones de la casa comercial. Esta prueba permite detectar anticuerpos específicos frente a Rotavirus Tipo A. La sensibilidad y especificidad reportada para este estuche comercial es de $97 \%$.

La interpretación de resultados para las pruebas de ELISA se realizó de acuerdo con las indicaciones de la casa productora, teniendo en cuenta las densidades ópticas (DO) tanto de los controles como de las muestras. Así, DO Control Positivo a 1/200: >0.7; DO Control Negativo a 1/200: $<0.4$; Punto de corte: 0.4 , donde muestras con valores superiores al punto de corte se consideraron positivas. La seroprevalencia (SP) fue calculada teniendo en cuenta la siguiente fórmula: $\mathrm{SP}=$ (Total de sueros positivos / Total de muestras) $* 100$. 
Cuadro 1. Seroprevalencia de rotavirus por grupo etario en granjas porcinas de ciclo completo del departamento de Cundinamarca, Colombia

\begin{tabular}{lccc}
\hline Fase de producción & $\begin{array}{c}\text { Muestras } \\
(\mathrm{n})\end{array}$ & $\begin{array}{c}\text { Muestras } \\
\text { positivas }(\mathrm{n})\end{array}$ & $\begin{array}{c}\text { Seroprevalencia } \\
(\%)\end{array}$ \\
\hline $\begin{array}{l}\text { Hembras (gestación- } \\
\text { lactancia-reemplazo) }\end{array}$ & 62 & 53 & 85.48 \\
Lechón lactante & 15 & 12 & 80.00 \\
Levante & 19 & 17 & 89.47 \\
Pre-cebo & 48 & 34 & 70.83 \\
Ceba & 23 & 15 & 65.22 \\
Machos & 3 & 3 & 100.0 \\
\hline Total & 170 & 134 & 78.82 \\
\hline
\end{tabular}

Cuadro 2. Seroprevalencia de rotavirus por granja porcícola de ciclo completo, según el municipio de procedencia (Cundinamarca, Colombia)

\begin{tabular}{|c|c|c|c|}
\hline \multirow{2}{*}{$\begin{array}{l}\text { Municipio } \\
\text { (ubicación de las granjas) }\end{array}$} & \multicolumn{2}{|c|}{ Muestras } & \multirow{2}{*}{$\begin{array}{c}\text { Seroprevalencia } \\
(\%)\end{array}$} \\
\hline & Por granja (n) & Positivas (n) & \\
\hline $\begin{array}{l}\text { Ricaurte } \\
\qquad\left(4^{\circ} 16^{\prime} 45^{\prime \prime} \mathrm{N} 74^{\circ} 46^{\prime} 22^{\prime \prime} \mathrm{O}\right)\end{array}$ & 10 & 10 & 100 \\
\hline $\begin{array}{l}\text { La Vega } \\
\quad\left(4^{\circ} 59^{\prime} 57^{\prime \prime} \mathrm{N} 74^{\circ} 20^{\prime} 28^{\prime \prime} \mathrm{O}\right)\end{array}$ & 11 & 9 & 81.81 \\
\hline $\begin{array}{l}\text { Nocaima } \\
\qquad\left(5^{\circ} 04^{\prime} 13^{\prime \prime} \mathrm{N} 74^{\circ} 22^{\prime} 41^{\prime \prime} \mathrm{O}\right)\end{array}$ & 11 & 10 & 90.91 \\
\hline $\begin{array}{l}\text { Zipaquirá } \\
\qquad\left(5^{\circ} 01^{\prime} 29^{\prime \prime} \mathrm{N} 74^{\circ} 00^{\prime} 05^{\prime \prime} \mathrm{O}\right)\end{array}$ & 40 & 29 & 72.5 \\
\hline $\begin{array}{l}\text { Sasaima } \\
\qquad\left(4^{\circ} 57^{\prime} 54^{\prime \prime} \mathrm{N} 74^{\circ} 26^{\prime} 05^{\prime \prime} \mathrm{O}\right)\end{array}$ & 40 & 26 & 65 \\
\hline $\begin{array}{l}\text { Bituima } \\
\quad\left(4^{\circ} 52^{\prime} 28^{\prime \prime} \mathrm{N} 74^{\circ} 32^{\prime} 26^{\prime \prime} \mathrm{O}\right)\end{array}$ & 58 & 50 & 86.21 \\
\hline Total & 170 & 134 & 78.82 \\
\hline
\end{tabular}

\section{Resultados}

El 78.82\% ( $\mathrm{n}=134)$ de las 170 muestras analizadas fueron positivas, encontrándose una mayor seroprevalencia en los machos, mientras los valores más bajos correspondieron a cerdos en la etapa de ceba (Cuadro 1). Los resultados de seropositividad de acuerdo con la granja estuvieron en un rango de $65-$ $100 \%$ (Cuadro 2). 
Discusión

La determinación serológica de la presencia de rotavirus en granjas porcícolas no es una práctica rutinaria, dado que no es un patógeno de reporte obligatorio. Aun así, en el presente estudio la seroprevalencia general fue de $78.82 \% \mathrm{y}$, aunque los reportes serológicos son escasos, los resultados obtenidos podrían ser compatibles con la prevalencia del 71.5\% reportada en Brasil con la técnica de RT-PCR (Médici et al., 2011); sin embargo, también por pruebas moleculares, se reportan prevalencias más bajas, en varios países europeos como Hungría, Dinamarca, España y Eslovenia $(4.2,10,16$ y 20\%, respectivamente), según Midgley et al. (2012), aunque sin hacer mención de los tipos de granja o a las medidas de bioseguridad instauradas en ellas.

Los Rotavirus se han asociado a procesos de enteritis y diarreas agudas en lechones lactantes y en destetos, e incluso en hembras lactantes (Médici et al., 2011; Vlasova et al., 2017); sin embargo, este virus también ha sido detectado en animales asintomáticos de diferentes edades (Miyazaki et al., 2013), lo que coincide con lo encontrado en el presente estudio en el que se evaluaron animales sin sintomatología gastrointestinal, a lo largo de la cadena primaria. Esta situación, no obstante, podría estar asociada con la inmunidad adquirida a través del calostro suministrado a los neonatos (Vlasova et al., 2017).

La presencia del rotavirus desencadena pérdidas económicas importantes, pues se reportan índices de mortalidad entre 7 y $20 \%$ en lechones lactantes y entre 3 y $15 \%$ en destetados (Shao et al., 2016). Por otro lado, el virus puede abrir la puerta a otros patógenos y pueden presentarse infecciones mixtas, por lo que, el monitoreo serológico contra este agente podría permitir la implementación de medidas de prevención y control tendientes al mejoramiento del estado sanitario de los animales.
Si bien, el potencial de transmisión zoonótica está reportado para los serogrupos A y $\mathrm{C}$, la presencia conjunta de los serotipos $\mathrm{G} 2,3,4,9,12$ y $\mathrm{P} 6,8$ en porcinos y en humanos, hace suponer la posible rotación entre especies hospederas (Martella et al., 2010; Midgley et al., 2012; Vlasova et al., 2017), especialmente a través de consumo de agua y alimentos contaminados (Kittigul et al., 2001). Adicionalmente, la presencia del virus en el agua (residual, de fuentes hídricas naturales, subterránea y potable) se debe a la contaminación de esta con materia fecal de individuos infectados (Kittigul et al. (2001) y que podrían entrar en contacto con otras cepas de rotavirus provenientes de diferentes especies hospederas, lo que implica un riesgo, pues el virus posee diferentes mecanismos para su reordenamiento genético (Martella et al., 2010). Se ha establecido que partículas de rotavirus reordenados, de origen humano y porcino, han sido capaces de infectar de forma exitosa poblaciones humanas de Latinoamérica (Alfieri et al., 1996), India (Varghese et al., 2004), Tailandia (Okada et al., 2000) e Italia (Martella et al., 2008).

\section{Conclusiones}

La presencia de anticuerpos contra rotavirus detectada en varios grupos etarios de granjas porcícolas de Cundinamarca, Colombia, sugiere que los animales evaluados pudieron estar en contacto con el virus en algún momento de su ciclo productivo, lo que no excluye la posibilidad de que los anticuerpos en cerdos lactantes y destetados se deriven de la inmunidad lactogénica transmitida por las cerdas.

\section{Agradecimientos}

Los autores agradecen a la Asociación Colombiana de Porcicultores (PorkColombia) por el apoyo económico para la obtención del estuche de diagnóstico, así como a los propietarios y técnicos de las granjas que parti- 
ciparon voluntariamente en el estudio y a la Vicerrectoría de Investigación de la Pontificia Universidad Javeriana por el apoyo a las actividades de Semillero de Enfermedades Infecciosas Veterinarias y Zoonosis (ID: PTA 00008609).

\section{Literatura Citada}

1. Alfieri AA, Leite JP, Nakagomi O, Kaga E, Woods PA, Glass RI, Gentsch JR. 1996. Characterization of human rotavirus genotype $\mathrm{P}[8] \mathrm{G} 5$ from Brazil by probe-hybridization and sequence. Arch Virol 141: 2353-2364. doi: 10.1007/ BF01718636

2. Blanco MA, Reyes D. 2015. Estadístico de la enfermedad diarreica aguda en pacientes atendidos en servicio de urgencias durante el 2012-2013. Rev Cuban Invest Bioméd 34: 104-111.

3. Bucardo F, Nordgren J. 2015. Impact of vaccination on the molecular epidemiology and evolution of group A rotaviruses in Latin America and factors affecting vaccine efficacy. Infect Genet Evol 34: 106-113. doi: 10.1016/j.meegid.2015.06.013

4. Casas G 2013. Protocolo toma de muestra de sangre en porcinos. Universidad Nacional de Colombia. [Internet]. Disponible en: http://medicinaveterinaria ydezootecnia.bogota.unal.edu.co/ fileadmin/FVMZ/Servicios/bioetica/ Pro_autorizados/002_Protocolo_toma_muestra_sangre_en_cerdos.pdf

5. [DANE] Departamento Administrativo Nacional de Estadística. 2019. Encuesta de sacrificio de ganado. [Internet]. Disponible en: www.dane.gov.co/index.php/estadisticas por tema/agropecuario/ encuesta de sacrificio-de-ganado

6. De la Hoz F, Alvis N, Narváez J, Cediel N, Gamboa O, Velandia M. 2010. Potential epidemiological and economical impact of two rotavirus vaccines in Colombia. Vaccine 28: 38563864. doi: 10.1016/j.vaccine.2010.03.004
7. Framstad T, Sjaastad Ø, Aass RA. 2000. Bleeding and intravenous techniques in pigs. Norwegian School of Veterinary Science. Oslo. [Internet]. Disponible en: http://oslovet.norecopa.no/ teaching/pig/pigbleed

8. Kittigul L, Khamoun P, Sujirarat D, Utrarachkij F, Chitpirom K, Chaichantanakit N, Vathanophas K. 2001. An improved method for concen-trating rotavirus from water samples. Mem Inst Oswaldo Cruz 96: 815-821. doi: 10.1590/ s0074-02762001000600013

9. Long CP, McDonald SM. 2012. Rotavirus genome replication: Some assembly required. PLoS Pathog 13: e1006242. doi: 10.1371/journal.ppat.1006242

10. Malasao R, Khamrin P, Kumthip K, Maneekarn N, Ushijima H. 2018. Complete genome sequence analysis of rare G4P[6] rotavirus strains from human and pig reveals the evidence for interspecies transmission. Infect Genet Evol 65:357-368. doi: 10.1016/j.meegid.2018.08.019

11. Martella V, Colombrita D, Lorusso E, Draghin E, Fiorentini S, De Grazia $S$, Bányai K, et al. 2008. Detection of a porcine-like rotavirus in a child with enteritis in Italy. J Clin Microbiol 46: 3501-3507. doi: 10.1128/JCM.00983-08

12. Martella V, Bányai K, Matthijnssens J, Buonavoglia C, Ciarlet M. 2010. Zoonotic aspects of rotaviruses. Vet Microbiol 140: 246-255. doi: 10.1016/ j.vetmic. 2009.08.028

13. Maya C. 2018. Sector porcicultor, uno de los más productivos del momento. [Internet]. Disponible en https:// www.dinero.com/edicion-impresa/negocios/articulo/balance-del-sectorporcicultor-en-colombia/255321

14. Médici KC, Barry AF, Alfieri AF, Alfieri AA. 2011. Porcine rotavirus groups $\mathrm{A}, \mathrm{B}$, and $\mathrm{C}$ identified by polymerase chain reaction in a fecal sample collection with inconclusive results by polyacrylamide gel electrophoresis. J Swine Health Prod 19: 146-150. 
15. Midgley SF, Banyai K, Buesa J, Halaihel N, Hjulsager ChK, Jakab F, Kaplon J. et al. 2012. Diversity and zoonotic potential of rotaviruses in swine and cattle across Europe. Vet Microbiol 156: 238-245. doi: 10.1016/j.vetmic.2011.10.027

16. Miyazaki A, Kuga K, Suzuki T, Kohmoto M, Katsuda K, Tsunemitsu H. 2013. Annual changes in predominant genotypes of rotavirus A detected in the feces of pigs in various developmental stages raised on a conventional farm. Vet Microbiol 163: 162-166. doi: 10.1016/ j.vetmic.2012.11.044

17. Monini M, Bartolo I, Ianiro G, Angeloni G, Magistrali ChF, Ostanello F, Ruggeri FM. 2015. Detection and molecular characterization of zoonotic viruses in swine fecal samples in Italian pig herds. Arch Virol 160: 2547-2556. doi: 10.1007/s00705-015-2538-4

18. Okada Ji, Urasawa T, Kobayashi N, Taniguchi K, Hasegawa A, Mise K, Urasawa S. 2000. New P serotype of group A human rotavirus closely related to that of a porcine rotavirus. J Med Virol 60: 63-69.

19. PorkColombia. 2014. Bioseguridad en granjas porcinas. Principios básicos e implementación en granjas porcícolas. [Internet]. Disponible en: https://repository.agrosavia.co/handle/20.500.12324/1991

20. Saurabh S, Sircar S, Kattoor JJ, Ghosh $S$, Kobayashi N, Banyai K, VinodhKumar OR, et al. 2018. Analysis of structure-function relationship in porcine rotavirus A enterotoxin gene. J Vet Sci 19: 35-43. doi: 10.4142/jvs.2018.-19.1.35

21. Shao L, Fischer DD, Kandasamy S, Rauf A, Langel SN, Saif LJ, et al. 2016. Comparative in vitro and in vivo studies of porcine rotavirus G9P[13] and human rotavirus Wa G1P[8]. J Virol 90: 142-151. doi: 10.1128/JVI.02401-15
22. Vlasova AN, Amimo JO, Saif LJ. 2017. Porcine rotaviruses: epidemiology, immune responses and control strategies. Viruses 9: E48. doi: 10.3390/v9030048

23. Vidal A, Clilverd H, Cortey M, Martín-Valls GE, Franzo G, Darwich L, Martín M, et al. 2018. Full-genome characte-rization by deep sequencing of rotavirus A isolates from outbreaks of neonatal diarrhoea in pigs in Spain. Vet Microbiol 227: 12-19. doi: 10.1016/ j.vetmic.2018.10.002

24. Varghese V, Das S, Singh NB, Kojima $K$, Bhattacharya SK, Krishnan T, Kobayashi N, et al. 2003. Molecular characterization of a human rotavirus reveals porcine characteristics in most of the genes including VP6 and NSP4. Arch Virol 149: 155-172. doi: 10.1007/ s00705-003-0199-1

25. [WHO] World Health Organization. 2017. Diarrhoeal disease. [Internet]. Disponible en: https://www.who.int/ news-room/fact-sheets/detail/diarrhoealdisease

26. Yang XL, Matthijnssens J, Sun H, Muhamaiti J, Zhang B, Nahar S, Van Ranst M, Rahman M. 2008. Temporal changes of rotavirus strain distribution in a city in the northwest of China, 19962005. Int J Infect Dis 12: 11-17. doi: 10.1016/j.ijid.2008.03.022

27. Yodmeeklin A, Khamrin P, Chuchaona $W$, Kumthip K, Kongkaew A, Vachirachewin R, Okitsu S, Ushijima H, Maneekarn N. 2017. Analysis of complete genome sequences of G9P[19] rotavirus strains from human and piglet with diarrhea provides evidence for whole-genome interspecies transmission of no reassorted porcine rotavirus. Infect Genet Evol 47: 99-108. doi: 10.1016/ j.meegid.2016.11.021 